\title{
A STABILITY RESULT FOR NONLINEAR NEUMANN PROBLEMS IN REIFENBERG FLAT DOMAINS IN $\mathbb{R}^{N}$
}

\author{
Antoine Lemenant and Emmanouil Milakis
}

\begin{abstract}
In this paper we prove that if $\Omega_{k}$ is a sequence of Reifenberg-flat domains in $\mathbb{R}^{N}$ that converges to $\Omega$ for the complementary Hausdorff distance and if in addition the sequence $\Omega_{k}$ has a "uniform size of holes", then the solutions $u_{k}$ of a Neumann problem of the form

$$
\begin{cases}-\operatorname{div} a\left(x, \nabla u_{k}\right)+b\left(x, u_{k}\right)=0 & \text { in } \Omega_{k} \\ a\left(x, \nabla u_{k}\right) \cdot \nu=0 & \text { on } \partial \Omega_{k}\end{cases}
$$

converge to the solution $u$ of the same Neumann problem in $\Omega$. The result is obtained by proving the Mosco convergence of some Sobolev spaces, that follows from the extension property of Reifenberg-flat domains.
\end{abstract}

\section{Introduction}

In this paper we study the stability of solutions for the following nonlinear Neumann problem

$$
\begin{cases}-\operatorname{div} a(x, \nabla u)+b(x, u)=0 & \text { in } \Omega \\ a(x, \nabla u) \cdot \nu=0 & \text { on } \partial \Omega\end{cases}
$$

where $\Omega$ is a bounded subset of $\mathbb{R}^{N}, a: \mathbb{R}^{N} \times \mathbb{R}^{N} \rightarrow \mathbb{R}^{N}$ and $b: \mathbb{R}^{N} \times \mathbb{R} \rightarrow \mathbb{R}$ are two Carathéodory functions satisfying suitable monotonicity, coerciveness and growth conditions (see (1.1)-(1.3) below). More precisely, we are interested in the following question. Let $\Omega_{k}$ be a sequence of open sets in $\mathbb{R}^{N}$ that converges to $\Omega$ for the complementary Hausdorff distance. Let $u_{k}$ be the sequence of solutions for the problem (0.2) in $\Omega_{k}$ and let $u$ be the solution associated to $\Omega$. Is it true that $u_{k}$ converges to $u$ ? If the answer is positive we say that the problem (0.2) is stable along the sequence $\Omega_{k}$ (see Definition 3).

2010 Mathematics Subject Classification. 35J65, 49Q20, 49J45.

Key words. Boundary value problems, nonlinear elliptic equations, Hausdorff distance, Reifenberg-flat sets, Mosco convergence. 
This question was studied by many authors in the last decade, principally in dimension 2 , for smooth and non smooth domains. The case of non smooth domain, and more specifically domains with cracks, appears typically in applications from fracture mechanics. In addition, the problem of stability is linked to the notions of Mosco convergence or Gamma convergence, which are powerful tools to study the semicontinuity of functionals in shape optimization problems or for instance the Mumford-Shah functional in image processing.

Recently, G. Dal Maso, F. Ebobisse and M. Ponsiglione [3] proved that in dimension 2 , if $\Omega_{k}$ tends to $\Omega$ for the complementary Hausdorff distance in $\mathbb{R}^{2},\left|\Omega_{k}\right|$ tends to $|\Omega|$ and if the number of the connected components of the complements $\Omega_{k}^{c}$ are uniformly bounded, then the problem $(0.2)$ is stable. This stability property extends the corresponding results of A. Chambolle and F. Doveri $[2]$ and also D. Bucur and $\mathrm{N}$. Varchon [1]. In particular, the authors showed that the stability is equivalent to the convergence of some Banach spaces in the sense of Mosco.

According to the authors knowledge, only few results have been proven in higher dimensions. In [8], A. Giacomini proves a stability result in $\mathbb{R}^{N}$ for fractured locally Lipschitz domains where an approach involving the Mosco convergence is also used.

A famous example called the "Neumann Sieve" ([7], [15], [17]), shows that in many cases the stability is not true. The general idea, for instance in dimension 2 , is that if one considers a sequence of 1-dimensional sets $E_{k}$ in $B(0,1)$ with more and more holes of size $\frac{1}{k}$ but with always same global length, it could happen that $\left\{E_{k}\right\}$ converges to a segment while the problem $(0.2)$ is not stable along the sequence $\left\{B(0,1) \backslash E_{k}\right\}$.

In this paper, we prove that if $\left\{\Omega_{k}\right\}$ converges to $\Omega$ for the complementary Hausdorff distance in $\mathbb{R}^{N}$ and if $\left\{\Omega_{k}\right\}$ is a sequence of Reifenberg flat domains with "uniform size of holes", then problem (0.2) is stable along the sequence $\left\{\Omega_{k}\right\}$ (see Theorem 7). The main point is to prove that the limit in the sense of Mosco, of the space of restrictions of functions in $W^{1, p}\left(\Omega_{k}\right)$ is the space of restrictions of functions in $W^{1, p}(\Omega)$.

The notion of "uniform size of holes" (Definition 8) is here to avoid the "Neumann Sieve" problem as described above. Indeed, we want to allow crack domains but according to our definition of uniform size of holes, the "tips" of the cracks can never become arbitrary closer to each other at the limit.

Our theorem partially extends the result of $[3]$ in any dimension, and it also can be considered as an extension of $[\mathbf{8}]$ since the regularity of our sets is weaker than Lipschitz. Indeed, Reifenberg flat sets are ones that 
are locally well-approximated by hyperplanes, at every scale. This allows for instance some "Hölderian spirals" or fractal boundaries (see [6]).

Reifenberg flat sets are rough domains that have naturally been used in the study of boundary regularity and the regularity of free boundaries coming from a minimization problem (see for instance [4], [5], [10], [12], [14] and references therein). As we shall see in the sequel, Reifenberg flat domains are moreover very well-adapted in the construction of good extensions for functions in the Sobolev space, which is the key ingredient for proving the Mosco convergence.

This sort of technics were also used in [11], and probably appeared for the first time in [9] while Peter Jones was seeking some geometrical conditions to define a class of extension domains. Notice however that our extension is slightly different than the classical extension of Jones, since we allow ourselves to modify the function inside the domain in a very small neighborhood of the boundary. On the other hand there is a little topological difficulty to overcome, coming from the fact that our domains admit some cracks (i.e. holes in the boundary).

Once the Extension Lemma is established, the Mosco convergence comes fairly easily and this completes the proof of our main result. Let us list here some notations that will be used along the present paper.

\section{Notation.}

$\partial \Omega:=\bar{\Omega} \backslash \Omega$, the topological boundary of $\Omega$.

$W^{1, p}(\Omega):=$ the Sobolev space.

$C_{c}^{1}(\Omega):=$ space of $C^{1}(\Omega)$ functions with compact support.

$\nu:=$ the outward normal vector.

$|A|:=$ the Lebesgue measure of the Borel set $A$.

$d_{H}:=$ the Hausdorff distance (defined in (1.7)).

$A^{c}:=$ the complement of the set $A$.

$A \triangle B:=A \backslash B \cup B \backslash A$.

$B(x, r):=$ the ball with center at $x$ and radius $r$.

$C:=$ a positive constant, that could vary from line to line, and that only could depend on dimension.

\section{Stability for the nonlinear Neumann problem}

Let $\Omega$ be an open bounded subset of $\mathbb{R}^{N}$ and let the real numbers $p$ and $q$ satisfy $1<p \leq 2 \leq q<+\infty$ and $\frac{1}{p}+\frac{1}{q}=1$. In this section 
we prove the stability for problem (0.2) where $a: \mathbb{R}^{N} \times \mathbb{R}^{N} \rightarrow \mathbb{R}^{N}$ and $b: \mathbb{R}^{N} \times \mathbb{R} \rightarrow \mathbb{R}$ are two Carathéodory functions satisfying the following assumptions: there exist $0<c_{1} \leq c_{2}, \alpha \in L^{q}\left(\mathbb{R}^{N}\right)$, and $\beta \in L^{1}\left(\mathbb{R}^{N}\right)$ such that, for almost every $x \in \mathbb{R}^{N}$ and for every $\xi, \xi_{1}, \xi_{2} \in \mathbb{R}^{N}$ with $\xi_{1} \neq \xi_{2}$

$$
\begin{aligned}
& \left\langle a\left(x, \xi_{1}\right)-a\left(x, \xi_{2}\right),\left(\xi_{1}-\xi_{2}\right)\right\rangle>0 ; \\
& |a(x, \xi)| \leq \alpha(x)+c_{2}|\xi|^{p-1} ; \\
& a(x, \xi) \cdot \xi \geq-\beta(x)+c_{1}|\xi|^{p} .
\end{aligned}
$$

We assume that $b$ satisfies the same inequalities, with possible different $\alpha$, $\beta, c_{1}$ and $c_{2}$. For simplicity we use the same notation.

$$
\begin{aligned}
& \left\langle b\left(x, \xi_{1}\right)-b\left(x, \xi_{2}\right),\left(\xi_{1}-\xi_{2}\right)\right\rangle>0 \\
& |b(x, \xi)| \leq \alpha(x)+c_{2}|\xi|^{p-1} \\
& b(x, \xi) \cdot \xi \geq-\beta(x)+c_{1}|\xi|^{p} .
\end{aligned}
$$

Throughout the paper we assume that $(0.2)$ is satisfied in the usual weak sense of Sobolev spaces, that is $u \in W^{1, p}(\Omega)$ and

$$
\int_{\Omega}\langle a(x, \nabla u), \nabla \varphi\rangle+\langle b(x, u), \varphi\rangle \mathrm{d} x=0
$$

for all $\varphi \in W^{1, p}(\Omega)$. It is well known that problem (0.2) has a unique solution in $W^{1, p}(\Omega)$ (see $\left.[\mathbf{1 3}]\right)$.

If $A$ and $B$ are two nonempty closed subsets of $\mathbb{R}^{N}$, we define the Hausdorff distance between $A$ and $B$ by

$$
d_{H}(A, B):=\max \left\{\sup _{x \in B} d(x, A), \sup _{x \in A} d(x, B)\right\}
$$

where $d(x, A)=\operatorname{dist}(x, A)$.

Definition 1. Let $\left\{\Omega_{k}\right\}_{k \in \mathbb{N}}$ and $\Omega$ be some nonempty open subsets of $\mathbb{R}^{N}$. We say that $\Omega_{k}$ converges to $\Omega$ for the complementary Hausdorff distance if $d_{H}\left(\Omega_{k}^{c}, \Omega^{c}\right)$ tends to 0 .

Remark 2. If $\Omega_{k}$ tends to $\Omega$ for the complementary Hausdorff distance, then $d_{H}\left(\partial \Omega_{k}, \partial \Omega\right)$ tends to zero, but observe that the converse is false in general (for instance take $\Omega_{k}:=\left(\frac{1}{k},+\infty\right)$ and $\Omega:=(-\infty, 0)$ ). 
Suppose that $\Omega_{k}$ converges to $\Omega$ for the complementary Hausdorff distance, and let $u_{k}$ be a weak solution of the problem

$$
\begin{cases}-\operatorname{div} a\left(x, \nabla u_{k}\right)+b\left(x, u_{k}\right)=0 & \text { in } \Omega_{k} \\ a\left(x, \nabla u_{k}\right) \cdot \nu=0 & \text { on } \partial \Omega_{k} .\end{cases}
$$

Definition 3. We say that problem (0.2) is stable along the sequence $\Omega_{k}$, if the following holds: Let $u_{k} \in W^{1, p}\left(\Omega_{k}\right)$ be a sequence of solutions of the problem (1.8) in $\Omega_{k}$. Then $\left(1_{\Omega_{k}} u_{k}, 1_{\Omega_{k}} \nabla u_{k}\right)$ converges strongly to $\left(1_{\Omega} v, 1_{\Omega} \nabla v\right)$ in $L^{p}\left(\mathbb{R}^{N}\right) \times L^{p}\left(\mathbb{R}^{N}, \mathbb{R}^{N}\right)$ and $v$ is a solution of problem $(0.2)$.

We seek conditions on $\Omega_{k}$ to make the problem (0.2) stable.

Definition 4. A $\left(\delta, r_{0}\right)$-Reifenberg-flat domain $\Omega \subset \mathbb{R}^{N}$ is an open bounded set such that for each $x \in \partial \Omega$ and for any $r \leq r_{0}$ there exist a hyperplane $P(x, r)$ containing $x$ such that

$$
\frac{1}{r} \sup _{y \in \partial \Omega \cap B(x, r)} \operatorname{dist}(y, P(x, r)) \leq \delta .
$$

Our definition of Reifenberg-flat domains is not exactly the same that could be found in the literature, essentially from the fact that we did not take a bilateral definition of the distance in (1.9) in order to allow cracks (when the domain lies in each side of its boundary). Using the terminology of [11], our Reifenberg-flat domains should be called "weak Reifenberg-flat domains". Observe that our definition allows the fact that $\partial \Omega$ could have an infinite number of connected components. Moreover in our definition, $\Omega$ is not supposed to be connected.

The topological disc theorem of Reifenberg [16] says that, under some additional separation conditions and if $\delta$ is small enough, then the boundary of a $\left(\delta, r_{0}\right)$-Reifenberg-flat domain is locally the bi-hölderian image of a $N-1$ dimensional unit disc. In addition, this is optimal since a Reifenberg-flat domain can admit some Hölder spiral. It is worth mentioning that a Reifenberg-flat domain could have a fractal "snowflakelike" boundary (see $[\mathbf{6}]$ ).

Now we consider a topological assumption in order to avoid the problem of "Neumann Sieves" (see [15]). For a Reifenberg-flat domain $\Omega$ and for any ball $B(x, r)$ centered at $\partial \Omega$ and with radius $r \leq r_{0}$, let us define the sets $D^{+}(x, r)$ and $D^{-}(x, r)$ by the following way. Let $P(x, r)$ be the hyperplane given by the definition of Reifenberg flatness of $\Omega$. Denote by $z^{ \pm}(x, r)$ two points of $B(x, r)$ that lie at distance $3 r / 4$ from $P(x, r)$ and whose orthogonal projections on $P(x, r)$ are equal to $x$. Then we set $D^{ \pm}(x, r):=B\left(z^{ \pm}, r / 4\right)$ as in the following picture. 


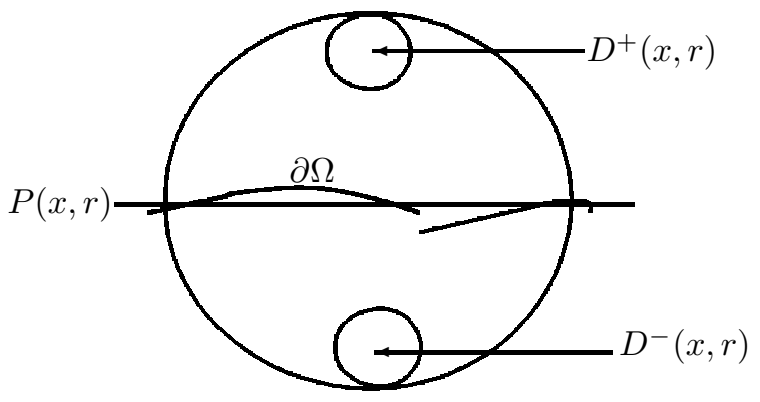

Definition 5. Let $\Omega$ be a $\left(\delta, r_{0}\right)$-Reifenberg flat domain. We say that $\Omega$ has a uniform size of holes with constant $C_{0} \geq 1 / 2$, if for every ball $B(x, r)$ centered at $\partial \Omega$ with radius $r \leq \frac{1}{2} r_{0}$ and such that $D^{+}(x, r)$ and $D^{-}(x, r)$ lie in the same connected component of $B(x, r) \cap \Omega$, there exists a ball $B(y, s)$ centered on $P(x, 2 r) \cap B(x, 2 r)$ with radius $s>\frac{1}{C_{0}} r$ such that $B(y, s) \cap \partial \Omega=\emptyset$. By convention if $\Omega$ is such that $D^{ \pm}(x, r)$ always lie in different connected components we say that $\Omega$ has a uniform size of holes with $C_{0}=1 / 3$.

Remark 6. If $\Omega$ has a uniform size of holes with constant $C_{0} \geq 1 / 2$ then $\Omega$ has a uniform size of holes with any constant $C>C_{0}$. Therefore, we will usually take $C_{0}$ as being the minimal constant satisfying the property of Definition 5 which can never be less than $1 / 2$ for obvious geometrical reasons. Moreover when we mean that a sequence $\Omega_{k}$ has a uniform size of holes with same constant $C_{0}$, we say that all the minimal constants associated to $\Omega_{k}$ are bounded by a same constant $C_{0}$ (see also the examples given below).

The rest of the section is devoted to the proof of the following result.

Theorem 7. Let $C_{0} \in[2,+\infty) \cup\{1 / 3\}$ be a constant and assume that $\left\{\Omega_{k}\right\}_{k \in \mathbb{N}}, \Omega$ are $\left(\delta, r_{0}\right)$-Reifenberg flat domains with $\delta<10^{-3} C_{0}^{-1}$, having a uniform size of holes with same constant $C_{0}$, and such that $\Omega_{k}$ converges to $\Omega$ for the complementary Hausdorff distance. Then the Neumann problem (0.2) is stable along the sequence $\Omega_{k}$.

Before passing to the details of the proof of Theorem 7, we give two examples for domains described in the discussion above. 


\subsection{Examples.}

We would like to emphasize the fact that according to Definition 5 , in Theorem 7 we do not consider "sieve domains" with holes in the boundary that are becoming smaller when $\Omega_{k}$ tends to $\Omega$, but only "crack domains" which contain some holes but with a fixed size bigger than $C_{0}^{-1} r_{0}$ and controlled shape. To illustrate this, let us show two basic examples in dimension 2 .

\subsubsection{A counterexample.}

Let us consider the 2 dimensional Sieve Domain defined as follow. For a fixed $\varepsilon$ set $r_{n}:=\varepsilon 2^{-n}$,

$$
\Gamma_{n}:=[0,1] \backslash \bigcup_{k=1}^{2^{n}}\left[k 2^{-n}-r_{n}, k 2^{-n}\right]
$$

and $\Omega_{n}:=B((1 / 2,0), 1) \backslash \Gamma_{n}$.

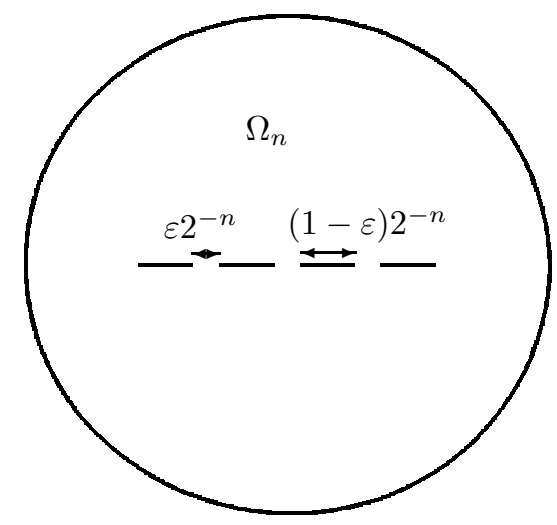

Since the boundary of $\Omega_{n}$ is "smooth", for every $\delta \in(0,1)$, one can easily obtain a radius $r_{\delta} \in\left(0, \frac{1}{10}\right)$ such that all the $\Omega_{n}$ are $\left(\delta, r_{\delta}\right)$-Reifenberg flat domains. Now for $n$ big enough, we claim that the minimal constant $C_{0}^{n}$ of uniform size of holes in $\Omega_{n}$ is bounded from below by $C 2^{n}$. Indeed, we denote $x_{0}:=(1 / 2,0) \in \mathbb{R}^{2}$ and consider the ball $B\left(x_{0}, r_{\delta} / 2\right)$. The corresponding domains $D^{ \pm}\left(x_{0}, r_{\delta} / 2\right)$ lie in the same connected components of $\Omega$ and $P\left(x, r_{\delta} / 2\right)$ is the first axis. Now since $r_{\delta}<\frac{1}{10}$, any ball $B(y, s)$ centered on $P\left(x_{0}, r_{\delta}\right) \cap B\left(x_{0}, r_{\delta}\right)$ and such that $B(y, s) \cap \partial \Omega_{n}=\emptyset$ must have a radius $s<\varepsilon 2^{-(n+1)}$. This means that

$$
C_{0}^{n} \geq r_{\delta} \frac{2^{n+1}}{\varepsilon}
$$


thus the sequence $\Omega_{n}$ cannot have a uniform size of hole with same constant $C_{0}$.

\subsubsection{An example with fractured domains.}

Further, we give another example where the domains $\Omega_{n}$ have now a uniform size of holes with same constant. Of course we could also take some "non-fractured" domains for which the constant is fixed to $\frac{1}{3}$ by convention, but let us consider the following more instructive crack situation. Let $S$ be the segment $[-1,1] \times\{0\} \subset \mathbb{R}^{2}$ and $\Omega:=B(0,2) \backslash S$. Now let $\Gamma_{n}:=\left\{\left(t, f_{n}(t)\right) ; t \in\left[-a_{n}, a_{n}\right] \subset \mathbb{R}\right\}$ be a sequence of Lipschitz graphs with same Lipschitz constant $L$ and such that $d_{H}\left(\Gamma_{n}, S\right) \leq$ $2^{-n-3}$

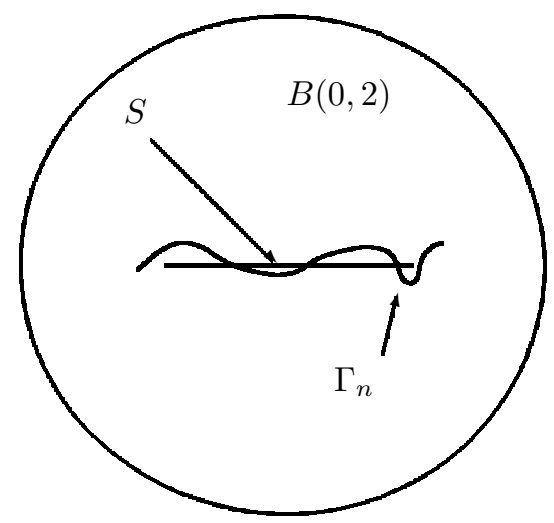

It is not difficult to see that the sequence $\Omega_{n}:=B(0,2) \backslash \Gamma_{n}$ is a sequence of $\left(\delta, r_{0}\right)$-Reifenberg-flat domains converging to $\Omega \backslash S$ for the complementary Hausdorff distance. Indeed, they are Reifenberg-flat with a good choice of $\delta$ and $r_{0}$ depending only on the Lipschitz constant $L$. Moreover we have that $d_{H}\left(\Omega_{n}^{c}, \Omega^{c}\right)=d_{H}\left(\Gamma_{n}, S\right) \leq 2^{-n-3}$. Finaly, $\Omega_{n}$ and $\Omega$ have all a uniform size of holes with constant $C_{0} \leq 10$. This is easy to prove for $\Omega$ because the only way for a ball $B(x, r)$ to be centered on $\partial \Omega$ and having the property that $B(x, r) \cap \Omega$ is connected is to be centered on $S$ with a radius $r>\operatorname{dist}(x, E(S))$ where $E(S)$ are the two endpoints of $S$. In this case it is clear that $B(x, 2 r)$ contains a ball of radius $s>10^{-1} r$ centered on the first axis that does not meet $\partial \Omega$. Now one can see this also for $\Omega_{n}$ by exactly the same argument, replacing the endpoints of $S$ by the endpoints of $\Gamma_{n}$, using also that $\Gamma_{n}$ is a graph. 
Of course in our example, Lipschitz graphs was assumed for convenience and one could try to weaken the regularity assumption on $\Gamma_{n}$ taking for instance a sequence of connected Reifenberg-flat sets with admitting some tangents at the endpoints, but the proof becomes a bit more technical in this case.

\subsection{Extension Lemma.}

The main ingredient for proving the Mosco convergence will be the Extension Lemma contained in this section. For every function $u \in$ $L^{1}(B(x, r))$ we denote by $m^{ \pm}(u)$ the average of $u$ on $D^{ \pm}(x, r)$

$$
m^{ \pm}(u):=\frac{1}{\left|D^{ \pm}(x, r)\right|} \int_{D^{ \pm}(x, r)} u(x) \mathrm{d} x .
$$

We begin with the following fact.

Proposition 8. Let $p \geq 1, C_{0} \geq 1 / 2$ and let $\Omega$ be a $\left(\delta, r_{0}\right)$-Reifenberg flat domain with $\delta<10^{-3} C_{0}^{-1}$ and having uniform size of holes with constant $C_{0}$. Then for every ball $B(x, r)$ centered at $\partial \Omega$ with $r \leq \frac{1}{4} r_{0}$ such that $D^{+}(x, r)$ and $D^{-}(x, r)$ lie in the same connected component of $B(x, r) \cap \Omega$, we have that

$$
\left|m^{+}(u)-m^{-}(u)\right| \leq C \frac{1}{r^{N-1}} \int_{B(x, 3 r)}|\nabla u| \mathrm{d} x
$$

for any function $u \in W^{1,1}(B(x, 3 r) \cap \Omega)$, and where $C$ is depending on dimension $N$ and constant $C_{0}$.

Proof: The proof is an easy consequence of the classical Poincaré inequality. Indeed, the definition of Uniform size of holes implies that there exist a ball $B(x, s)$ centered on $P(x, 2 r) \cap B(x, 2 r)$ such that $B(x, s) \cap \partial \Omega=\emptyset$, and with $s>\frac{1}{C_{0}} r$. Now the proposition follows from the following fact: since $\partial \Omega$ is at distance less than $2 \delta r<2.10^{-3} C_{0}^{-1} r$ from $P(x, 2 r)$ in $B(x, 2 r)$, one can define a Lipschitz domain $A$ contained in $B(x, 3 r)$, that contains $D^{+}(x, r) \cup D^{-}(x, r) \cup B\left(x, \frac{s}{2}\right)$, and such that the Poincaré constant in $A$ is less than $C\left(C_{0}, N\right) r$, where $C\left(C_{0}, N\right)$ is only depending on $C_{0}$ and $N$ and is independent from all the possible positions of $B(x, s)$.

Next we give the Extension lemma. 
Lemma 9. Let $r_{0}$ be a positive radius, $\tau \in\left(0, r_{0}\right)$ and $C_{0} \in[1 / 2,+\infty) \cup$ $\{1 / 3\}$. Let $\Omega_{1}$ and $\Omega_{2}$ be two $\left(\delta, r_{0}\right)$-Reifenberg-flat domains with $\delta<$ $10^{-3} C_{0}^{-1}$, having a uniform size of holes with same constant $C_{0}$ and such that

Then setting

$$
d_{H}\left(\Omega_{1}^{c}, \Omega_{2}^{c}\right) \leq 10^{-3} C_{0}^{-1} \tau
$$

$$
W(\tau):=\left\{y \in \mathbb{R}^{N} ; d\left(y, \partial \Omega_{1}\right) \leq \tau\right\},
$$

for any $v \in W^{1, p}\left(\Omega_{1}\right)$ there exists a function $\tilde{v} \in W^{1, p}\left(\Omega_{2}\right)$ such that $v=\tilde{v}$ in $\Omega_{1} \backslash W(10 \tau)$ and

$$
\begin{aligned}
\|\tilde{v}\|_{L^{p}\left(\Omega_{2} \cap W(10 \tau)\right)} & \leq C\|v\|_{L^{p}\left(\Omega_{1} \cap W(10 \tau)\right)} \\
\|\nabla \tilde{v}\|_{L^{p}\left(\Omega_{2} \cap W(10 \tau)\right)} & \leq C_{1}\|\nabla v\|_{L^{p}\left(\Omega_{1} \cap W(100 \tau)\right)}
\end{aligned}
$$

with $C$ depending only on the dimension $N$, while $C_{1}$ depends on constant $C_{0}$ and on dimension $N$.

Proof: Let $\tau \in\left(0, r_{0}\right)$ be fixed and let $B_{i}:=B\left(x_{i}, \tau\right)$ be a family of balls of radius $\tau$, centered at $x_{i} \in \partial \Omega_{1}$, and maximal for the property that $\frac{1}{10} B_{i} \cap \frac{1}{10} B_{j}=\emptyset$ for all $i, j \in I$ with $i \neq j$. Notice that by this way,

$$
W(9 \tau) \subset \bigcup_{i \in I} 10 B_{i} \subset W(10 \tau) .
$$

We want to construct a partition of unity associated to $\left\{B_{i}\right\}_{i \in I}$. For all $i$, define a function $\varphi_{i} \in C_{c}^{1}\left(10 B_{i}\right)$, such that $\varphi=1$ in $8 B_{i},|\nabla \varphi| \leq \tau^{-1}$ and let $\varphi_{0}$ be a function that is equal to 1 in $\Omega_{1} \backslash \bigcup_{i \in I} 10 B_{i}, \varphi_{0}=0$ in $\bigcup_{i \in I} 8 B_{i}$ and $\varphi_{0}+\sum_{i \in I} \varphi_{i} \geq 1$ in $\Omega_{1} \cup \bigcup_{i \in I} 10 B_{i}$. Moreover, we can assume that there is a constant $C$ such that for all $x \in 10 B_{i} \backslash 8 B_{i}$, $\left|\nabla \varphi_{0}(x)\right| \leq C \tau^{-1}$. Indeed, such a function $\varphi_{0}$ can be obtained by setting

$$
\varphi_{0}(x):=\prod_{i \in I} l\left(d\left(x, x_{i}\right) / \tau\right)
$$

where $l$ is a Lipschitz function equal to 0 in $[0,8]$, equal to 1 in $[10,+\infty)$ and $l^{\prime}(x) \leq 10$. Finally, define

$$
\theta_{i}:=\frac{\varphi_{i}}{\varphi_{0}+\sum_{i \in I} \varphi_{i}} \quad \text { for } i \in I \cup\{0\}
$$

thus we now have a partition of unity in $\Omega_{1} \cup \bigcup_{i \in I} B_{i}$. To define a function $\tilde{v} \in W^{1, p}\left(\Omega_{2}\right)$, it suffices to define an extension of $v$ in $\Omega_{2} \cap$ $\bigcup_{i \in I} 10 B_{i}$ since $\Omega_{1} \triangle \Omega_{2}$ is contained in $\bigcup_{i \in I} 10 B_{i}$.

Let $P_{i}:=P\left(x_{i}, 10 \tau\right)$ be the hyperplane associated to $\Omega_{1}$ given by Definition 4. Recall that since $d_{H}\left(\Omega_{1}^{c}, \Omega_{2}^{c}\right) \leq 10^{-3} C_{0}^{-1} \tau$, we have that $10 B_{i} \cap\left(\partial \Omega_{2}\right) \subset\left\{y \in 10 B_{i} ; d\left(y, P_{i}\right) \leq 2.10^{-2} C_{0}^{-1} \tau\right\}$. 
For each ball $B_{i}$, we denote by $D^{ \pm}\left(x_{i}, 10 \tau\right)$ the two balls defined just before Definition 8 associated to $\Omega_{1}$, and we assume this notation to be coherent with the orientation of $\partial \Omega_{1}$. More precisely we assume the notation of positive and negative signs of $D^{ \pm}\left(x_{i}, 10 \tau\right)$, to be chosen in such a way that for any $i, j$ satisfying $B\left(x_{i}, 10 \tau\right) \cap B\left(x_{j}, 10 \tau\right) \neq \emptyset$, we have that $D^{+}\left(x_{i}, 10 \tau\right)$ and $D^{+}\left(x_{j}, 10 \tau\right)$ both lie in the same connected component of $B\left(x_{i}, 100 \tau\right) \backslash P\left(x_{i}, 100 \tau\right)$. This of course can be done without loss of generality, starting from a first choice of $D^{+}\left(x_{j}, 10 \tau\right)$ and then defining the others successively. Then we need to consider three cases.

- Cracktip case: $D^{+}\left(x_{i}, 10 \tau\right)$ and $D^{-}\left(x_{i}, 10 \tau\right)$ lie both in the same connected component of $B(x, 10 \tau) \cap \Omega_{2}$. Let $I_{c}$ be the set of indices corresponding to the balls in this situation and for every $i \in I_{c}$ define $m_{i}:=m^{+}(v)$ (as in (1.10)).

- Boundary case 1: $D^{+}\left(x_{i}, 10 \tau\right)$ and $D^{-}\left(x_{i}, 10 \tau\right)$ lie in different connected components of $B(x, 10 \tau) \cap \Omega_{2}$. Let $I_{b_{1}}$ be the set of indices corresponding to the balls in this situation. For every $i \in$ $I_{b_{1}}$, let $A_{i}^{+}$and $A_{i}^{-}$be the two connected components of $10 B_{i} \backslash \partial \Omega_{2}$ that contain respectively $D^{+}\left(x_{i}, 10 \tau\right)$ and $D^{-}\left(x_{i}, 10 \tau\right)$ and define $m_{i}^{ \pm}:=m^{ \pm}(v)$.

- Boundary case 2: One of $D^{ \pm}\left(x_{i}, 10 \tau\right)$ lies in $\Omega_{2}$ while the other one lies in $\Omega_{2}^{c}$. Let $I_{b_{2}}$ be the set of indices corresponding to the balls in this situation. For every $i \in I_{b_{2}}$, let $m_{i}$ be equal to the one of $m^{ \pm}(v)$ corresponding to ball $D^{ \pm}\left(x_{i}, 10 \tau\right)$ that lies in $\Omega_{2}$. Finally as for $I_{b_{1}}$, let $A_{i}^{+}$and $A_{i}^{-}$be the two connected components of $10 B_{i} \backslash \partial \Omega_{2}$ that contain respectively $D^{+}\left(x_{i}, 10 \tau\right)$ and $D^{-}\left(x_{i}, 10 \tau\right)$.

Notice that since $\tau$ is chosen sufficiently small, the preceding 3 cases would be the same if $\Omega_{1}$ was taken instead of $\Omega_{2}$ in their definition.

We are now ready to define $\tilde{v}$. It could be that $\Omega_{2}$ has some tiny connected components hidden in some $10 B_{i} \backslash\left(A_{i}^{+} \cup A_{i}^{-}\right)$for $i \in I_{b_{1}}$. In those components, let us define $\tilde{v}$ to be equal to 0 . Then, anywhere else, i.e. for all $x \in A:=\left(\Omega_{2} \backslash \bigcup_{i \in I_{b_{1}}} 10 B_{i}\right) \cup\left(\bigcup_{i \in I_{b_{1}}} A_{i}^{+} \cup A_{i}^{-}\right)$, define

$$
\tilde{v}(x):=\theta_{0} v(x)+\sum_{i \in I_{c} \cup I_{b_{2}}} \theta_{i}(x) m_{i}+\sum_{i \in I_{b_{1}}} \theta_{i}(x)\left(m_{i}^{+} 1_{A_{i}^{+}}(x)+m_{i}^{-} 1_{A_{i}^{-}}(x)\right) .
$$

The function $\tilde{v}$ is now well defined for every $x \in \Omega_{2}$. We claim that $\tilde{v} \in W^{1, p}\left(\Omega_{2}\right)$ and that (1.12) and (1.13) are satisfied. Let us first show (1.12). Using Hölder inequality and extending $v$ by 0 outside of $\Omega_{1}$, 
we have for all $i \in I_{b_{2}} \cup I_{c}$,

$$
\left|m_{i}\right|^{p} \leq C\left(\frac{1}{\left|B_{i}\right|} \int_{B_{i}}|v| \mathrm{d} x\right)^{p} \leq C\left|B_{i}\right|^{-1} \int_{B_{i}}|v|^{p}
$$

and by the same way for $i \in I_{b_{1}}$ we also have

$$
\left|m_{i}^{ \pm}\right|^{p} \leq C\left|B_{i}\right|^{-1} \int_{B_{i}}|v|^{p} .
$$

In addition, since $B_{i}$ are in a bounded cover (with a universal constant $C)$, the sums in (1.14) are locally finite. Thus, since $\theta_{i}(x) \leq$ $1_{10 B_{i}}(x)$ and using (1.15) and (1.16),

$$
\begin{aligned}
\left\|\sum_{i \in I_{c} \cup I_{b_{2}}} \theta_{i}(x) m_{i}\right\|_{L^{p}\left(\Omega_{2}\right)}^{p} & \leq C \sum_{i \in I_{c} \cup I_{b_{2}}} \int_{10 B_{i}}\left|m_{i}\right|^{p} \\
& \leq C \sum_{i \in I} \int_{10 B_{i}}|v|^{p} \mathrm{~d} x \leq C\|v\|_{L^{p}(W(10 \tau))}^{p}
\end{aligned}
$$

and by the same way

$$
\begin{aligned}
\left\|\sum_{i \in I_{b_{1}}} \theta_{i}(x)\left(m_{i}^{+} 1_{A_{i}^{+}}(x)+m_{i}^{-} 1_{A_{i}^{-}}(x)\right)\right\|_{L^{p}\left(\Omega_{2}\right)}^{p} & \leq C \sum_{i \in I} \int_{10 B_{i}}|v|^{p} \mathrm{~d} x \\
& \leq C\|v\|_{L^{p}(W(10 \tau))}^{p}
\end{aligned}
$$

thus taking the $L^{p}$ norm in (1.14) we deduce that (1.12) holds. Let us now prove (1.13), which will also imply that $\tilde{v} \in W^{1, p}\left(\Omega_{2}\right)$. By definition, $\tilde{v}=v$ in $\Omega_{2} \backslash \bigcup_{i \in I} 10 B_{i}$, thus all we have to prove is that

$$
\int_{A \cap \bigcup_{i \in I} 10 B_{i}}|\nabla \tilde{v}|^{p} \leq \int_{\Omega_{1} \cap W(60 \tau)}|\nabla v|^{p} .
$$

Let $i_{0}$ be a fixed index such that $x \in A \cap 10 B_{i_{0}}$. Let $I_{x}$ be the finite set of indices $i \in I$ such that $x \in B_{i}$. All balls $B_{i}$ for $i \in I_{x}$ are contained in $B\left(x_{i_{0}}, 20 \tau\right)$. Let us first make the following assumption on the orientation:

$$
\begin{aligned}
& \text { For any } i \in\left(I_{b_{1}} \cup I_{b_{2}}\right) \cap I_{x}, x \text { lie in the same connected } \\
& \text { component as } D\left(x_{i_{0}}, 10 \tau\right)^{+} \text {of } B\left(x_{i}, 10 \tau\right) \cap \Omega_{2} .
\end{aligned}
$$

Next we define a convex domains $D_{x}^{+}$, as being the convex hull of all the $D^{+}\left(x_{i}, 10 \tau\right)$ for $i \in I_{x}$. Since $\left(\partial \Omega_{1} \cup \partial \Omega_{2}\right) \cap B\left(x_{i_{0}}, 20 \tau\right)$ is contained in $\left\{y ; d\left(y, P_{0}\right) \leq \frac{2 \tau}{10}\right\}$ for some hyperplane $P_{0}$, we have that $D_{x}^{+}$does not meet $\left(\partial \Omega_{1} \cup \partial \Omega_{2}\right) \cap B\left(x_{i_{0}}, 20 \tau\right)$ and is contained in $B\left(x_{i_{0}}, 100 \tau\right)$. 
Define

$$
\begin{array}{ll}
m_{x}^{+}:=\frac{1}{\left|D_{x}^{+}\right|} \int_{D_{x}^{+}} v(x) \mathrm{d} x, & \text { and } \\
m_{i_{0}} & :=\frac{1}{\left|D\left(x_{i_{0}}, 20 \tau\right)^{+}\right|} \int_{D\left(x_{i_{0}}, 20 \tau\right)^{+}} v(x) \mathrm{d} x .
\end{array}
$$

Since $\nabla \theta_{0}+\sum_{i \in I} \nabla \theta_{i}=0$ we have, using our assumption (1.18),

$$
\begin{aligned}
\nabla \tilde{v}(x)=\theta_{0}(x) \nabla v(x) & +\nabla \theta_{0}(x) v(x)+\sum_{i \in I_{c} \cup I_{b_{2}}} \nabla \theta_{i} m_{i}+\sum_{i \in I_{b_{1}}} \nabla \theta_{i} m_{i}^{+} 1_{A_{i}^{+}}(x) \\
=\theta_{0}(x) \nabla v(x) & +\underbrace{\nabla \theta_{0}(x)\left(v(x)-m_{i_{0}}\right)}_{f_{1}} \\
& +\underbrace{\sum_{i \in I_{c} \cup I_{b_{2}}} \nabla \theta_{i}\left(m_{i}-m_{i_{0}}\right)+\sum_{i \in I_{b_{1}}} \nabla \theta_{i}\left(m_{i}^{+}-m_{i_{0}}\right) .}_{f_{2}}
\end{aligned}
$$

To estimate $f_{1}$, since $\operatorname{supp}\left(\nabla \theta_{0}\right) \cap B\left(x_{i_{0}}, 60 \tau\right)$ is contained in a Lipschitz domain with universal constant, we infer that

$$
\begin{aligned}
\int_{B\left(x_{i_{0}}, 10 \tau\right)}\left|f_{1}(x)\right|^{p} & \leq \frac{1}{\tau^{p}} \int_{B\left(x_{i_{0}}, 60 \tau\right)} 1_{\operatorname{supp}\left(\nabla \theta_{0}(x)\right)}\left|v(x)-m_{i_{0}}\right|^{p} \\
& \leq C \int_{B\left(x_{i_{0}}, 60 \tau\right)}|\nabla v|^{p}
\end{aligned}
$$

Now we estimate $f_{2}$ still using the Poincaré inequality. Firstly for any $i \in I_{x} \cap I_{b_{1}}$ we have that

$$
\left|m_{i}^{+}-m_{i_{0}}\right| \leq\left|m_{i}^{+}-m_{x}^{+}\right|+\left|m_{x}^{+}-m_{i_{0}}\right| \leq \frac{C}{\tau^{N-1}} \int_{D_{x}^{+}}|\nabla v| .
$$

If $i \in I_{c}$, we have the same inequality with $m_{i}$ instead of $m_{i}^{+}$, because by definition $m_{i}=m^{+}(v)$ in this case.

Therefore we have proved that under assumption (1.18),

$$
\left|f_{2}(x)\right| \leq C \frac{1}{\tau^{N}} \int_{B\left(x_{i_{0}}, 100 \tau\right)}|\nabla v| \mathrm{d} x .
$$

Now assume that instead of (1.18) we had:

$$
\begin{aligned}
& \text { For any } i \in\left(I_{b_{1}} \cup I_{b_{2}}\right) \cap I_{x}, x \text { lie in the same connected } \\
& \text { component as } D\left(x_{i_{0}}, 10 \tau\right)^{-} \text {of } B\left(x_{i}, 10 \tau\right) \cap \Omega_{2} .
\end{aligned}
$$


Then we can proceed exactly as above with negative signs in the notations instead of positive signs, except for inequality (1.20) with $i \in I_{c}$ since by convention in this case we have set $m_{i}:=m^{+}(v)$. However, since the boundary of $\partial \Omega_{1}$ has a uniform size of holes, we can estimate the difference $\left|m^{+}(v)-m^{-}(v)\right|$ by (1.11). Indeed, we know that $D_{\Omega_{1}}^{ \pm}\left(x_{i}, 10 \tau\right)$ lie in the same connected component of $B\left(x_{i}, 10 \tau\right) \cap \Omega_{2}$. This means that there is a ball $B\left(y_{i}, s\right)$ centered on $P\left(x_{i}, 10 \tau\right) \cap B\left(x_{i}, 20 \tau\right)$ with radius $s>C_{0}^{-1} 20 \tau$, such that $B\left(y_{i}, s\right) \cap \partial \Omega_{1}=\emptyset$. But since $d_{H}\left(\Omega_{1}^{c}, \Omega_{2}^{c}\right)<$ $10^{-3} C_{0}^{-1} \tau$, we deduce that $B\left(y_{i}, \frac{s}{2}\right) \cap \partial \Omega_{2}=\emptyset$ thus $D_{\Omega_{2}}^{ \pm}\left(x_{i}^{\prime}, 20 \tau\right)$ (the one associated to $\Omega_{2}$ for a $x_{i}^{\prime} \in \partial \Omega_{2}$ satisfying $\left.\operatorname{dist}\left(x_{i}, x_{i}^{\prime}\right) \leq 10^{-3} C_{0}^{-1} \tau\right)$, lie also in the same connected components of $B\left(x_{i}^{\prime}, 20 \tau\right) \cap \Omega_{2}$. Then Proposition 8 , together with an other application of the Poincaré inequality to estimate the difference between the average on $D_{\Omega_{2}}^{ \pm}\left(x_{i}^{\prime}, 20 \tau\right)$ and the average on $D_{\Omega_{1}}^{ \pm}\left(x_{i}, 10 \tau\right)$, gives

$$
\left|m_{i}-m_{x}^{-}\right| \leq\left|m_{i}-m^{-}(v)\right|+\left|m^{-}(v)-m_{x}^{-}\right| \leq \frac{C}{\tau^{N-1}} \int_{B\left(x_{i}, 60 \tau\right)}|\nabla u|
$$

where $C$ is now depending on $C_{0}$.

Therefore, in any cases we have proved (1.23). Subsequently,

$$
\left\|f_{2}(x)\right\|_{L^{p}\left(B\left(x_{i_{0}}, 10 \tau\right)\right)} \leq\|\nabla v\|_{L^{p}\left(B\left(x_{i_{0}}, 100 \tau\right)\right)} .
$$

In conclusion we have obtained,

$$
\|\nabla \tilde{v}(x)\|_{L^{p}\left(\Omega_{2} \cap 10 B_{i_{0}}\right)} \leq C\|\nabla v\|_{L^{p}\left(\Omega_{1} \cup 100 B_{i_{0}}\right)},
$$

and (1.13) follows using the fact that $\left\{B_{i}\right\}$ forms a locally finite covering of $W(10 \tau)$.

\subsection{Mosco-convergence.}

For every open set $\Omega \subset \mathbb{R}^{N}$ we define the closed linear subspace $X_{\Omega}$ of $L^{p}\left(\mathbb{R}^{N}\right) \times L^{p}\left(\mathbb{R}^{N}, \mathbb{R}^{N}\right)$ by

$$
X_{\Omega}:=\left\{\left(u 1_{\Omega}, \nabla u 1_{\Omega}\right) ; u \in W^{1, p}(\Omega)\right\} .
$$

Definition 10 (Mosco-convergence). Let $\Omega_{k}$ and $\Omega$ be open subsets of $\mathbb{R}^{N}$ and let $X_{\Omega_{k}}$ and $X_{\Omega}$ be the corresponding subspaces of $L^{p}\left(\mathbb{R}^{N}\right) \times$ $L^{p}\left(\mathbb{R}^{N}, \mathbb{R}^{N}\right)$ defined by $(1.24)$. We say that $X_{\Omega_{k}}$ converges to $X_{\Omega}$ in the sense of Mosco if the following two properties hold:

$(M 1)$ for every $u \in W^{1, p}(\Omega)$, there exists a sequence $u_{k} \in W^{1, p}\left(\Omega_{k}\right)$ such that $u_{k} 1_{\Omega_{k}}$ converges to $u 1_{\Omega}$ strongly in $L^{p}\left(\mathbb{R}^{N}\right)$ and $\nabla u_{k} 1_{\Omega_{k}}$ converges to $\nabla u 1_{\Omega}$ strongly in $L^{p}\left(\mathbb{R}^{N}, \mathbb{R}^{N}\right)$; 
(M2) if $h_{k}$ is a sequence of indices converging to $\infty, u_{k}$ is a sequence such that $u_{k} \in W^{1, p}\left(\Omega_{h_{k}}\right)$ for every $k$, and $u_{k} 1_{\Omega_{h_{k}}}$ converges weakly in $L^{p}\left(\mathbb{R}^{N}\right)$ to a function $\phi$, while $\nabla u_{k} 1_{\Omega_{h_{k}}}$ converges weakly in $L^{p}\left(\mathbb{R}^{N}, \mathbb{R}^{N}\right)$ to a function $\psi$, then there exists $u \in W^{1, p}(\Omega)$ such that $\phi=u 1_{\Omega}$ and $\psi=\nabla u 1_{\Omega}$ a.e. in $\mathbb{R}^{N}$.

The Mosco convergence is a great tool to study stability for Neumann problems. In particular, we have the following result coming from $[\mathbf{3}$, Theorem 2.3], which is stated in $\mathbb{R}^{2}$ in [3] but can be extended in $\mathbb{R}^{N}$ with the same proof.

Theorem $11([3])$. Let $\Omega_{k}$ and $\Omega$ be open subsets of $\mathbb{R}^{N}$. Then $\Omega$ is stable for the problems $(0.2)$ along the sequence $\Omega_{k}$, if and only if $X_{\Omega_{k}}$ converges to $X_{\Omega}$ in the sense of Mosco.

According to Theorem 11, Theorem 7 will be a consequence of the following result.

Theorem 12. Let $r_{0}>0, C_{0} \in[1 / 2,+\infty) \cup\{1 / 3\}$ and let $\left\{\Omega_{k}\right\}_{k \in \mathbb{N}}$ and $\Omega$ be $\left(\delta, r_{0}\right)$-Reifenberg flat domains with $\delta<10^{-3} C_{0}^{-1}$ and having a uniform size of holes with same constant $C_{0}$. Assume that $\Omega_{k}$ converges to $\Omega$ for the complementary Hausdorff distance. Then $X_{\Omega_{k}}$ converges to $X_{\Omega}$ in the sense of Mosco.

Proof: Let $u \in W^{1, p}(\Omega)$ and assume without loss of generality that $\tau_{k}:=$ $d_{H}\left(\Omega_{k}^{c}, \Omega^{c}\right)<C_{0}^{-1} 10^{-4} r_{0}$. Set $W(t):=\{x ; d(x, \partial \Omega) \leq t\}$ and let $\tilde{u}_{k}$ be the extension function given in Lemma 9 for $\Omega_{1}:=\Omega, \Omega_{2}:=\Omega_{k}$, $v:=u, \tau:=C_{0} 10^{3} \tau_{k}$. Since $\tilde{u}_{k} \in W^{1, p}\left(\Omega_{k}\right)$, all we have to prove is that $\left(\tilde{u}_{k} 1_{\Omega_{k}}, \nabla \tilde{u}_{k} 1_{\Omega_{k}}\right)$ converges strongly to $\left(u 1_{\Omega}, \nabla u 1_{\Omega}\right)$ in $L^{p}\left(\mathbb{R}^{N}\right) \times$ $L^{p}\left(\mathbb{R}^{N}, \mathbb{R}^{N}\right)$. Since $u=\tilde{u}_{k}$ in $\Omega \backslash W\left(10 \tau_{k}\right)$ we have that

$$
\begin{aligned}
\left(\int_{\mathbb{R}^{N}}\left|\tilde{u} 1_{\Omega_{k}}-u 1_{\Omega}\right|^{p} \mathrm{~d} x\right)^{\frac{1}{p}} & =\left(\int_{W\left(10 \tau_{k}\right)}\left|\tilde{u} 1_{\Omega_{k}}-u 1_{\Omega}\right|^{p} \mathrm{~d} x\right)^{\frac{1}{p}} \\
& \leq\left(\int_{W\left(10 \tau_{k}\right)}\left|\tilde{u} 1_{\Omega_{k}}\right|^{p} \mathrm{~d} x\right)^{\frac{1}{p}}+\left(\int_{W\left(10 \tau_{k}\right)}\left|u 1_{\Omega}\right|^{p} \mathrm{~d} x\right)^{\frac{1}{p}} \\
& \leq C\|u\|_{L^{p}\left(W\left(10 \tau_{k}\right)\right)}
\end{aligned}
$$

which tends to zero when $k$ tends to $+\infty$ because $\partial \Omega$ is closed and $|\partial \Omega|=$ 0 . For the gradients, a similar argument can be done, using (1.13). That 
is,

$$
\begin{aligned}
\left(\int_{\mathbb{R}^{N}}\left|\nabla \tilde{u} 1_{\Omega_{k}}-\nabla u 1_{\Omega}\right|^{p} \mathrm{~d} x\right)^{\frac{1}{p}} & =\left(\int_{W\left(10 \tau_{k}\right)}\left|\nabla \tilde{u} 1_{\Omega_{k}}-\nabla u 1_{\Omega}\right|^{p} \mathrm{~d} x\right)^{\frac{1}{p}} \\
\leq & \left(\int_{W\left(10 \tau_{k}\right)}\left|\nabla \tilde{u} 1_{\Omega_{k}}\right|^{p} \mathrm{~d} x\right)^{\frac{1}{p}} \\
& +\left(\int_{W\left(10 \tau_{k}\right)}\left|\nabla u 1_{\Omega}\right|^{p} \mathrm{~d} x\right)^{\frac{1}{p}} \\
\leq & C\|\nabla u\|_{L^{p}\left(W\left(60 \tau_{k}\right)\right)}
\end{aligned}
$$

which tends to 0 , thus $(M 1)$ is proved.

Let us now prove $(M 2)$. Let $\varphi \in C^{\infty}\left(\mathbb{R}^{N}\right)$ be compactly supported in $\Omega$. Then we know by the weak convergence that

$$
\left\{\begin{array}{l}
\int_{\Omega} u_{k} 1_{\Omega_{h_{k}}} \varphi \mathrm{d} x \stackrel{k \rightarrow+\infty}{\longrightarrow} \int_{\Omega} \phi \varphi \mathrm{d} x \\
\int_{\Omega}\left\langle\nabla u_{k} 1_{\Omega_{h_{k}}}, \varphi\right\rangle \mathrm{d} x \stackrel{k \rightarrow+\infty}{\longrightarrow} \int_{\Omega} \psi \varphi \mathrm{d} x .
\end{array}\right.
$$

On the other hand, since $\Omega_{h_{k}}$ converges to $\Omega$ for the complementary Hausdorff distance, for $k$ large enough $1_{\Omega_{h_{k}}}$ is equal to 1 everywhere on the support of $\phi$. Thus (1.25) shows that $u_{k}$ converges to $\phi$ in $\mathcal{D}^{\prime}(\Omega)$ and $\nabla u_{k}$ converges to $\psi$ in $\mathcal{D}^{\prime}(\Omega)$. By uniqueness of the limit in $\mathcal{D}^{\prime}(\Omega)$ we conclude that

$$
\psi=\nabla \phi \quad \text { in } \quad \mathcal{D}^{\prime}(\Omega)
$$

Moreover since $\psi \in L^{p}\left(\mathbb{R}^{N}, \mathbb{R}^{N}\right)$, we deduce that (1.26) in true in $L^{p}\left(\Omega, \mathbb{R}^{N}\right)$ thus $\psi=\nabla \phi$ a.e. in $\Omega$ and therefore $\left.\phi\right|_{\Omega} \in W^{1, p}(\Omega)$. To conclude, all we have to show is that $\varphi=\psi=0$ in $\Omega^{c}$. To see this, we use a similar argument as above by defining a function $\varphi$ compactly supported in $\Omega^{c}$. By the weak convergence, and because $\Omega_{k}$ converges to $\Omega$ for the complementary Hausdorff distance, we deduce that $\int_{\Omega} \phi \varphi \mathrm{d} x=0$. This holds for any function $\varphi$ compactly supported in $\Omega^{c}$. Since $\phi \in L^{p}\left(\mathbb{R}^{N}\right)$ we conclude that $\phi=0$ in $\Omega^{c}$. In a similar way we obtain that $\psi=0$ in $\Omega^{c}$ and since the Lebesgue measure of $\partial \Omega$ is zero, the proof is complete. 


\section{Stability for problems with mixed boundary conditions}

In this last section we state some similar stability result for the problem with mixed boundary conditions, directly coming from the proof of Theorem 6.3 in [3]. The main motivation is that those statements could be useful to obtain estimates of solutions for elliptic partial differential equations in Reifenberg-flat domains using a perturbation argument.

Let $A \subset \mathbb{R}^{N}$ be a bounded open set with Lipschitz boundary $\partial A$, and let $\partial_{D} A$ be a relatively open subset of $\partial A$. For every compact set $K \subset \bar{A}$, for every $g \in W^{1, p}(A)$, and for every pair of function $a$ and $b$ satisfying the properties (1.1)-(1.3) we consider the solutions $u$ of the mixed problem

$$
\begin{cases}-\operatorname{div} a(x, \nabla u)+b(x, u)=0 & \text { in } A \backslash K, \\ u=g & \text { on } \partial_{D} A \backslash K, \\ a(x, \nabla u) \cdot \nu=0 & \text { on } \partial(A \backslash K) \backslash\left(\partial_{D} A \backslash K\right) .\end{cases}
$$

Definition 13. We say that the problem (2.1) is stable along the sequence $\left(K_{k}, g_{k}\right)$, if the following holds: Let $u_{k} \in W^{1, p}(A \backslash K)$ be a sequence of solutions of the problem (2.1) corresponding to $K_{k}$ and $g_{k}$. Then $\left(u_{k} 1_{A \backslash K_{k}}, \nabla u_{k} 1_{A \backslash K_{k}}\right)$ converges strongly to $\left(v 1_{A \backslash K}, \nabla v 1_{A \backslash K}\right)$ in $L^{p}\left(\mathbb{R}^{N}\right) \times L^{p}\left(\mathbb{R}^{N}, \mathbb{R}^{N}\right)$ and $v$ is a solution of problem (2.1).

For all $g \in W^{1, p}(A)$ we denote

$$
W_{g}^{1, p}\left(A \backslash K, \partial_{D} A \backslash K\right):=\left\{u \in W^{1, p}(A \backslash K): u=g \text { on } \partial_{D} \backslash K\right\} .
$$

As in (1.24) we define the closed linear subspace $X_{K}^{g}(A)$ of $L^{p}(A) \times$ $L^{p}\left(A, \mathbb{R}^{N}\right)$ by

$$
X_{K}^{g}(A):=\left\{\left(u 1_{K^{c}}, \nabla u 1_{K^{c}}\right) ; u \in W_{g}^{1, p}\left(A \backslash K, \partial_{D} A \backslash K\right)\right\} .
$$

Definition 14 (Mosco-convergence). We say that $X_{K_{k}}^{g_{k}}(A)$ converges to $X_{K}^{g}(A)$ in the sense of Mosco if the following two properties hold:

$\left(M 1^{\prime}\right)$ for every $u \in W_{g}^{1, p}\left(A \backslash K, \partial_{D} A \backslash K\right)$, there exists a sequence $u_{k} \in$ $W_{g_{k}}^{1, p}\left(A \backslash K_{k}, \partial_{D} A \backslash K_{k}\right)$ such that $u_{k} 1_{K_{k}^{c}}$ converges to $u 1_{K^{c}}$ strongly in $L^{p}(A)$ and $\nabla u_{k} 1_{K_{k}^{c}}$ converges to $\nabla u 1_{K^{c}}$ strongly in $L^{p}\left(A, \mathbb{R}^{N}\right)$;

$\left(M 2^{\prime}\right)$ if $h_{k}$ is a sequence of indices converging to $\infty, u_{k}$ is a sequence such that $u_{k} \in W_{g_{k}}^{1, p}\left(A \backslash K_{k}\right)$ for every $k$, and $u_{k} 1_{K_{k}^{c}}$ converges weakly in $L^{p}(A)$ to a function $\phi$, while $\nabla u_{k} 1_{K_{k}^{c}}$ converges weakly in $L^{p}\left(A, \mathbb{R}^{N}\right)$ to a function $\psi$, then there exists $u \in W_{g}^{1, p}\left(A \backslash K, \partial_{D} A \backslash K\right)$ such that $\phi=u 1_{K^{c}}$ and $\psi=\nabla u 1_{K^{c}}$ a.e. in $\mathbb{R}^{N}$. 
Remark 15. As it is pointed out in [3], by adopting the same proof as in Theorem 11, the Mosco convergence of $X_{K_{k}}^{g_{k}}(A)$ to $X_{K}^{g}(A)$ is equivalent to the stability of the mixed problem $(2.1)$ along the sequence $\left(K_{k}, g_{k}\right)$.

Finally we obtain the corresponding result in the case of the mixed boundary conditions.

Theorem 16. Let $A$ be a bounded open subset of $\mathbb{R}^{N}$ with Lipschitz boundary $\partial A$, let $\partial_{D} A$ be a relatively open subset of $\partial A$ and let $C_{0}$ be a positive constant. Let $g_{h}$ be a sequence in $W^{1, p}(A)$ converging strongly to a function $g \in W^{1, p}(A)$, and let $K_{h}$ be a sequence of compact subsets of $\bar{A}$ converging to a set $K$ in the Hausdorff metric. Assume in addition that for every $h, A \backslash K_{h}$ is a $\left(\delta, r_{0}\right)$-Reifenberg flat domain with $\delta<10^{-3} C_{0}^{-1}$ and having a uniform size of holes with constant $C_{0}$. Then $X_{K_{h}}^{g_{h}}(A)$ converges to $X_{K}^{g}(A)$ in the sense of Mosco.

Proof: The proof is essentially the same as the proof of Theorem 6.3 in [3], consisting of an idea due to Chambolle. The only difference is that the sets

$$
\Omega_{h}:=\Sigma \backslash\left(K_{h} \cup\left(\partial A \backslash \partial_{D} A\right)\right) \text { and } \quad \Omega:=\Sigma \backslash\left(K \cup\left(\partial A \backslash \partial_{D} A\right)\right)
$$

where $\Sigma$ is an open ball in $\mathbb{R}^{N}$ such that $\bar{A} \subset \Sigma$, are now satisfying the assumptions of Theorem 12 and thus the Mosco convergence in that setting. We refer the reader to [3, Theorem 6.3], for the details in that approach.

\section{Acknowledgements}

The second author was supported by the Marie Curie International Reintegration Grant No 256481 within the 7th European Community Framework Programme. Part of this work was done while the first author was affiliated with the research center Ennio De Giorgi and he wishes to thank all the members of the center and the Scuola Normale Superiore of Pisa for their hospitality. He also wishes to thank Tatiana Toro for the invitation in the University of Washington, where this work was initiated.

\section{References}

[1] D. Bucur And N. VArchon, Boundary variation for a Neumann problem, Ann. Scuola Norm. Sup. Pisa Cl. Sci. (4) 29(4) (2000), $807-821$. 
[2] A. Chambolle and F. Doveri, Continuity of Neumann linear elliptic problems on varying two-dimensional bounded open sets, Comm. Partial Differential Equations 22(5-6) (1997), 811-840.

[3] G. Dal Maso, F. Ebobisse, and M. Ponsiglione, A stability result for nonlinear Neumann problems under boundary variations, J. Math. Pures Appl. (9) 82(5) (2003), 503-532.

[4] G. DAVID, Hölder regularity of two-dimensional almost-minimal sets in $\mathbb{R}^{n}$, Ann. Fac. Sci. Toulouse Math. (6) 18(1) (2009), 65-246.

[5] G. David, T. De Pauw, and T. Toro, A generalization of Reifenberg's theorem in $\mathbb{R}^{3}$, Geom. Funct. Anal. 18(4) (2008), 1168-1235.

[6] G. David And T. Toro, Reifenberg flat metric spaces, snowballs, and embeddings, Math. Ann. 315(4) (1999), 641-710.

[7] T. Del Vecchio, The thick Neumann's sieve, Ann. Mat. Pura Appl. (4) 147 (1987), 363-402.

[8] A. Giacomini, A stability result for Neumann problems in dimension $N \geq 3, J$. Convex Anal. 11(1) (2004), 41-58.

[9] P. W. Jones, Quasiconformal mappings and extendability of functions in Sobolev spaces, Acta Math. 147(1-2) (1981), 71-88.

[10] A. Lemenant, Sur la régularité des minimiseurs de Mumford-Shah en dimension 3 et supérieure, Thesis University Paris-Sud XI Orsay (2008).

[11] A. Lemenant, Energy improvement for energy minimizing functions in the complement of generalized Reifenberg-flat sets, Ann. Sc. Norm. Super. Pisa Cl. Sci. (5) 9(2) (2010), 351-384.

[12] A. Lemenant and E. Milakis, Quantitative stability for the first Dirichlet eigenvalue in Reifenberg flat domains in $\mathbb{R}^{N}$, J. Math. Anal. Appl. 364(2) (2010), 522-533.

[13] J.-L. Lions, "Quelques méthodes de résolution des problèmes aux limites non linéaires", Dunod, Gauthier-Villars, Paris, 1969.

[14] E. Milakis And T. Toro, Divergence form operators in Reifenberg flat domains, Math. Z. 264(1) (2010), 15-41.

[15] F. Murat, The Neumann sieve, in: "Nonlinear variational problems" (Isola d'Elba, 1983), Res. Notes in Math. 127, Pitman, Boston, MA, 1985, pp. 24-32.

[16] E. R. Reifenberg, Solution of the Plateau Problem for $m$-dimensional surfaces of varying topological type, Acta Math. 104 (1960), $1-92$. 
[17] E. SÁnchez-PALEncia, Boundary value problems in domains containing perforated walls, in: "Nonlinear partial differential equations and their applications", Collège de France Seminar, Vol. III (Paris, 1980/1981), Res. Notes in Math. 70, Pitman, Boston, Mass.London, 1982, pp. 309-325.

Antoine Lemenant:

Université Paris Diderot - Paris 7

U.F.R. de Mathématiques

Site Chevaleret Case 7012

75205 Paris Cedex 13

France

E-mail address: lemenant@ann.jussieu.fr

Emmanouil Milakis:

University of Cyprus

Department of Mathematics \& Statistics

P.O. Box 20537

Nicosia, CY-1678

Cyprus

E-mail address: emilakis@ucy.ac.cy

Primera versió rebuda el 14 de setembre de 2010, darrera versió rebuda el 26 de novembre de 2010 . 